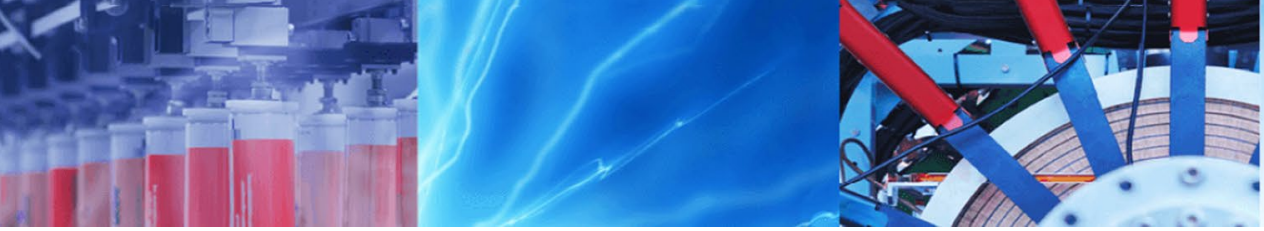

Research Article

\title{
Lifetime of electrochromic optical transition cycling of ethyl viologen diperchlorate-based electrochromic devices
}

\author{
Logan G. Kiefer ${ }^{1} \cdot$ Christian J. Robert $^{1} \cdot$ Taylor D. Sparks $^{1}$ (i)
}

Received: 16 September 2020 / Accepted: 1 April 2021

Published online: 13 April 2021

The Author(s) 2021 OPEN

\begin{abstract}
Electrochromic materials and devices are enabling a variety of advanced technologies. Gel-based organic electrochromic molecules such as ethyl viologen diperchlorate are attractive options due to their simple device design and low cost processing options relative to the more expensive and complex transition metal oxide films. However, electrochromic devices are subject to extensive cycling in which failure and fatigue can eventually occur. This work presents the lifetime cycling performance of ethyl viologen diperchlorate-based electrochromic devices using two different anodic compounds, hydroquinone and ferrocene, which are cycled at different voltages, $3 \mathrm{~V}$ and $1.2 \mathrm{~V}$, respectively. Multiple devices are cycled until failure with periodic device characterization via UV-Vis spectroscopy, electrical resistance and power measurement, and transition duration measurement. Devices with hydroquinone can transition quickly. Cycle times are $\sim 30 \mathrm{~s}$ in these samples, however, these samples also typically fail before 3000 cycles. On the other hand, devices using ferrocene transition more slowly (total cycle time $\sim 2 \mathrm{~min}$ ), but have superior cycling performance with all samples surviving beyond 10,000 cycles while complying with ASTM E2141-14 standard.
\end{abstract}

Keywords Lifetime $\cdot$ Electrochromism $\cdot$ Viologen $\cdot$ Cycling $\cdot$ Wearable electronics

\section{Introduction}

An electrochromic material's optical absorbance changes reversibly in response to an externally applied electric potential [1]. These electrochromic (EC) changes transition between a transparent ("bleached") state and a colored state, a property with many practical applications. This material's versatility has led to the development of a variety of EC devices. These devices include anti-glare car rearview mirrors, light-reflective or light-transmissive devices for optical information and storage, sunglasses, sports goggles, protective eye wear for the military, adjustable aircraft windows, glare-reduction systems for offices, display panels, and "smart windows" for use in buildings and automobiles [1-7].
Bipyridinium salts (viologens), a type of organic EC material, display different colors corresponding to their oxidation state and the nature of nitrogen's chemical bond's upon EC transition [8]. Figure 1 illustrates two viologen redox states. The dication is the most stable and is colorless.

Reductive electron transfer to viologen dications forms radical cations, which are colored. Suitable choices of nitrogen substituents in viologens to attain the appropriate molecular orbital energy levels can, in principle, allow color choice of the radical cation. For example, simple alkyl groups promote a blue/violet color whereas aryl groups generally impart a green hue to the radical cation [10]. The expensive processing of commercially available metaloxide EC materials, such as $\mathrm{WO}_{3}$, typically involves a highvacuum sputtering process. On the other hand, viologen

Taylor D. Sparks, sparks@eng.utah.edu | ${ }^{1}$ Materials Science and Engineering Department, University of Utah, Salt Lake City, UT, USA. 


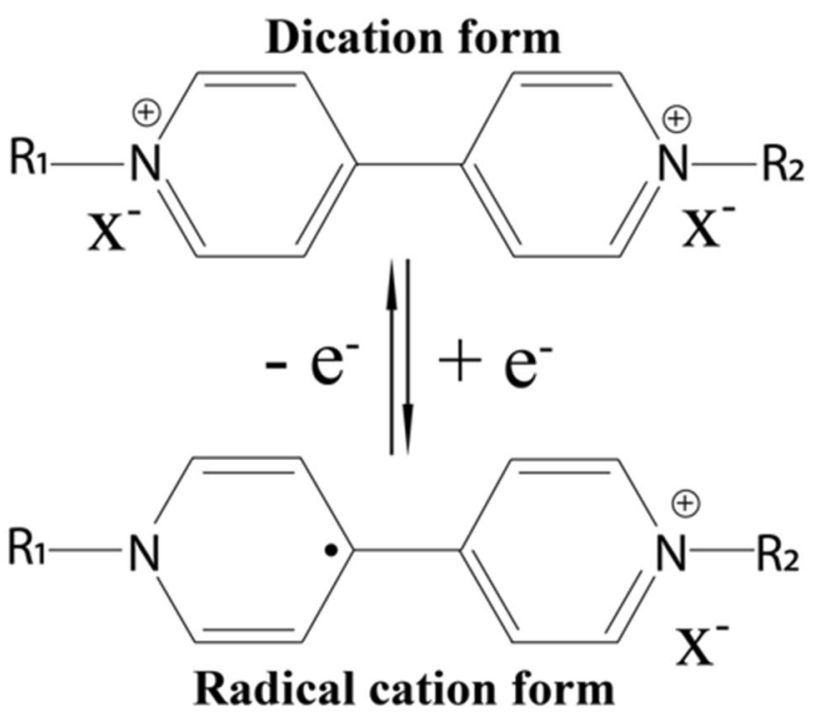

Fig. 1 Electrochemical transitions of viologen molecules. The dication form is colorless and most stable but can be reduced to the radical cation form which has a colored state (adapted from reference [9])

EC technology relies on low-cost non-vacuum manufacturing making it attractive for many practical applications.

With such a wide range of applications, it is important to understand the longevity of these EC devices. ASTM E2141 defines failure in electrochromic devices as when they achieve a Photoptic Transmittance Ratio (PTR) less than 4 [11]. PTR is defined as the percent transmittance in the bleached state at $550 \mathrm{~nm}$ divided by the percent transmittance in the colored state at $550 \mathrm{~nm}, \operatorname{PTR}=\tau_{b} / \tau_{c}$. One study described two different ethyl viologen diperchlorate (EV) based electrochromic devices that could be driven at different voltages [12]. They hypothesized that the devices that could be driven with a lower potential could be cycled for longer. This hypothesis is supported by findings in other viologen-based systems. [13]

This study addresses the longevity of these two different EV EC devices, by measuring the optical transmission through EC films, the absorbance of the EC films, the current through the EC films, the voltage across the EC films, and the cycling times during cycling.

\section{Materials and methods}

\subsection{EC device preparation}

Two different device types were prepared with different electrochromic viologen gel compositions first suggested by Chidichimo and coworkers [12]. For detailed device performance including cyclic voltammetry and coloration
Table 1 Amount of EC gel used in each cycling sample

\begin{tabular}{lll}
\hline & $\begin{array}{l}\text { PMMA/PC/EV/Hq } \\
\text { Gel amount }(\mathrm{g})\end{array}$ & $\begin{array}{l}\mathrm{PMMA} / \mathrm{PC} / \mathrm{EV} / \mathrm{FC} \\
\text { Gel amount }(\mathrm{g})\end{array}$ \\
\hline Sample 1 & 0.215 & 0.278 \\
Sample 2 & 0.231 & 0.295 \\
Sample 3 & 0.228 & 0.253 \\
Sample 4 & 0.214 & 0.271 \\
Sample 5 & Damaged & 0.282 \\
\hline
\end{tabular}

efficiency, we refer the reader to this original publication and instead report on cycling lifetime performance here. The first composition was prepared using using $0.89 \mathrm{wt} \%$ ethyl viologen diperchlorate (EV, MilliporeSigma, 98\% purity), $0.34 \mathrm{wt} \%$ hydroquinone ( $\mathrm{Hq}$, MilliporeSigma, $\geq$ 99\% purity), $16.49 \mathrm{wt} \%$ poly methyl methacrylate (PMMA, MilliporeSigma, crystalline, average $M_{w} \sim 996,000 \mathrm{~g} / \mathrm{mol}$ by GPC), and 82.28 wt\% propylene carbonate (PC, MilliporeSigma, $99.7 \%$ purity). This composition will be referred to as PMMA/PC/EV/HQ. The second composition was prepared using using $1.26 \mathrm{wt} \%$ ethyl viologen diperchlorate (MilliporeSigma, 98\% purity), $2.51 \mathrm{wt} \%$ ferrocene (FC, MilliporeSigma $98 \%), 18.69 \mathrm{wt} \%$ poly methyl methacrylate (MilliporeSigma, crystalline, average $M_{w} \sim 996,000 \mathrm{~g} / \mathrm{mol}$ by GPC), and $77.54 \mathrm{wt} \%$ propylene carbonate (MilliporeSigma, $99.7 \%$ purity). This composition will be referred to as PMMA/PC/EV/Fc. To prepare the EC devices, chemicals were weighed, combined, heated to $100^{\circ} \mathrm{C}$, and stirred thoroughly to obtain a homogeneous mixture. After cooling, a small portion of the solidified mixture was scooped and applied to the center of a $1.5^{\prime \prime} \times 1.5^{\prime \prime} 60 \Omega$ /square indium tin oxide (ITO) coated 7.0 mil polyethylene terephthalate (PET) substrate (Multek). Five samples of each composition were fabricated although Sample 5 of PMMA/ $\mathrm{PC} / \mathrm{EV} / \mathrm{Hq}$ was physically damaged due to handling during testing and was not included in analysis. Table 1 shows the amount of gel used for each sample at each composition. Another ITO-coated PET was placed above the gel to form a sandwich structure and this device was then pressed between two preheated aluminum plates $\left(80^{\circ} \mathrm{C}\right)$ and vacuum pressed using a Seal-A-Meal ${ }^{\mathrm{TM}}$ Vacuum Sealer (FSSMSL0160-000) for approximately $30 \mathrm{~s}$. Thin layers of plastic along two edges of the samples were used as spacers to obtain uniform thicknesses of approximately $0.4 \mathrm{~mm}$. 24 gauge copper wires (Striveday ${ }^{\mathrm{TM}}$ ) soldered to $0.25^{\prime \prime}$ copper conducting tape $\left(3 \mathrm{M}^{\mathrm{TM}} \mathrm{Code} 1182\right)$ were placed along both edges of the EC samples to make contact with the ITO layer. 


\subsection{Absorbance measurement methods}

The electro-optical properties of the EC device, the current through the device, the voltage across the device, and the ambient temperature were measured during cycling using a custom prepared microcontroller (Sparkfun electronics RedBoard). Photocell values were taken during cycling as a function of time. An RGB LED (China Young Sun LED Technology Co., Model No.: YSL-R596CR3G4B5CC10) was used as the light source. The relative transmittance of the sample was measured using a photocell (Lida Optical \& Electronic Co., Model No.: GL5528). The ambient temperature was measured using a digital temperature sensor (Analog Devices, Model No.: TMP36). The current was measured using a Keithley 2000 Multimeter (IEEE-488). The LED and the photocell were isolated from ambient light using copper tubing. The EC device was powered
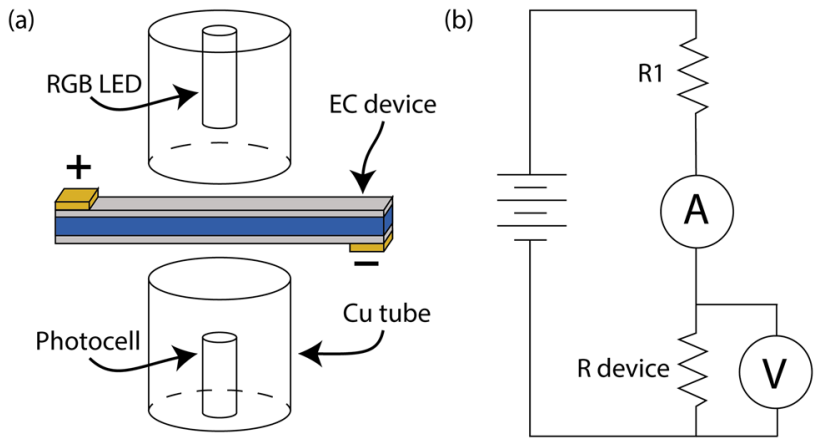

Fig. 2 a Transmittance data collection experimental setup, and b the schematic of current measurements through sample and voltage measurements across sample by two electrodes clipped to the copper wire contacts on each side of the sample. For the PMMA/PC/EV/Hq samples a voltage of approximately $3 \mathrm{~V}$ was used to drive the EC reaction, whereas a voltage of approximately $1.2 \mathrm{~V}$ was used to drive the PMMA/PC/EV/FC EC reactions. The lower applied voltage for the second EC gel composition was achieved using a voltage divider in series with the EC sample of $(480 \Omega)$. The custom prepared experimental setup is shown in Fig. 2 a.

Optical spectroscopy measurements of the EC samples were taken in both the bleached and colored states before cycling, after 10 cycles, after 100 cycles, after 1,000 cycles, after 10,000 cycles or after failure using a PerkinElmer UV/VIS/NIR Spectrometer Lambda 950 with the $150 \mathrm{~mm}$ Integrating Sphere attachment. The wavelength range of light scanned was from $250 \mathrm{~nm}$ to $1100 \mathrm{~nm}$ at a scan speed of $273.55 \mathrm{~nm} / \mathrm{min}$. Two sheets of ITO coated PET were used for the background scan. Cycling time was measured as the optical switching speed calculated as the time necessary to achieve $90 \%$ of the entire transmittance modulation.

\section{Resistance and power calculation}

Figure $2 \mathrm{~b}$ shows the schematic of how the current through the sample and the voltage across the sample were taken during cycling. Figure $3 a$ shows the voltage across the sample and the current through the sample during coloration. These values are changing which implies that the resistance of the device is changing with time. Using Ohm's law, the resistance of the device was determined during coloration, as shown at various different cycles in Fig. 3b,
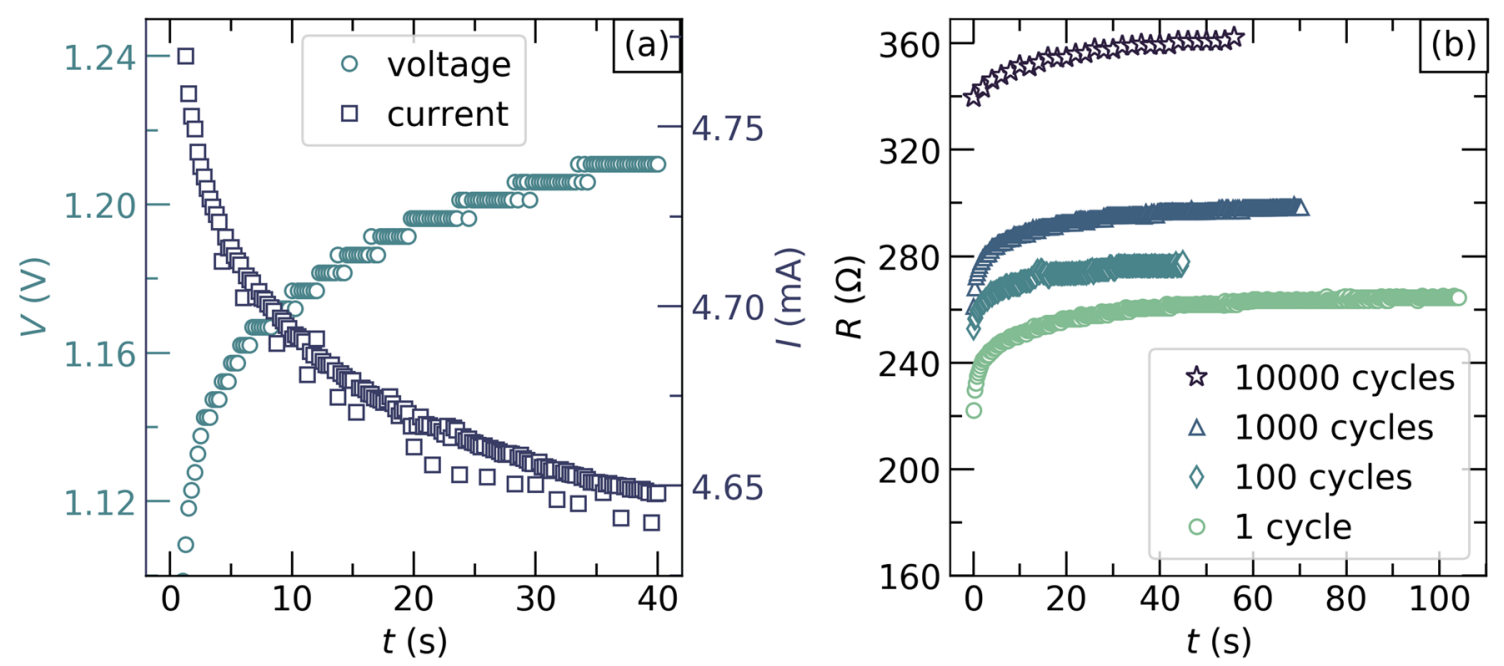

Fig. 3 a Voltage and current values for the coloration of Sample 1 during the hundredth cycle and, b computed PMMA/PC/EV/Fc Sample 1 resistance values during coloration at cycle 1, cycle 1001 cycle 1000, and cycle 10,000 
by dividing the voltage across the sample by the current through the sample. To determine the power required to drive the $\mathrm{EC}$ reaction, the voltage across the sample was multiplied by the current through the sample, as specified by the equation for power. These calculations assume that the ammeter has a relatively small input impedance, and that the voltmeter has a relatively high input impedance.

\section{Results and discussion}

\subsection{PMMA/PC/EV/Hq}

Figure 4a shows the cycle length as a function of cycle number for the overall cycle, coloring, and bleaching respectively. The overall cycle length remained relatively constant during the earlier part of the sample lifetime, and increased drastically before failure. The coloring length exhibited similar trends, drastically increasing before device failure at around cycle 2750 . However, bleaching length increased relatively steadily throughout the lifetime of the device.

Figures $5 \mathrm{a}, \mathrm{c}$ shows the UV-Vis spectroscopy transmittance data for Sample 3 at various different cycles in the bleached and colored states respectively. The percent transmittance in the bleached state seems to increase for visible light during cycling, suggesting that the sample becomes more transparent in the bleached state with more cycling. In the colored state the sample appears to achieve lower percent transmittance values with continued cycling until failure.

Figure 5 illustrates how the maximum absorbance at $605 \mathrm{~nm}$ changes with cycling and suggests that the absorbance of the samples in the colored state at this wavelength increases during the useful lifetime of the samples, and then plummets at failure. Achievable transmittance increases with increasing temperature.

PTR values were computed for all four samples at failure. Sample 1 failed at 3215 cycles with a PTR value of 4.26, Sample 2 failed at 1457 cycles with a PTR value of 7.40, Sample 3 failed at 1733 cycles with a PTR value of 2.31 , and Sample 4 failed at 2896 cycles with a PTR value of 1.95. Although the PTR values of Samples 1 and 2 were higher than the ASTM 2141 defined PTR for failure, qualitative analysis deems them unfit for continued use as they exhibit severe discoloration in the bleached state and nonuniform color in the colored state. These effects may be mitigated and EC samples will likely last longer by using $\mathrm{Fc}$ as the anodic compound, as opposed to $\mathrm{Hq}$, such that samples can be cycled at lower voltages.

\subsection{PMMA/PC/EV/FC}

The cycle length of the PMMA/PC/EV/Fc electrochromic samples is shown in Fig. 4b. While the total cycle time and coloring cycle time show no statistically significant

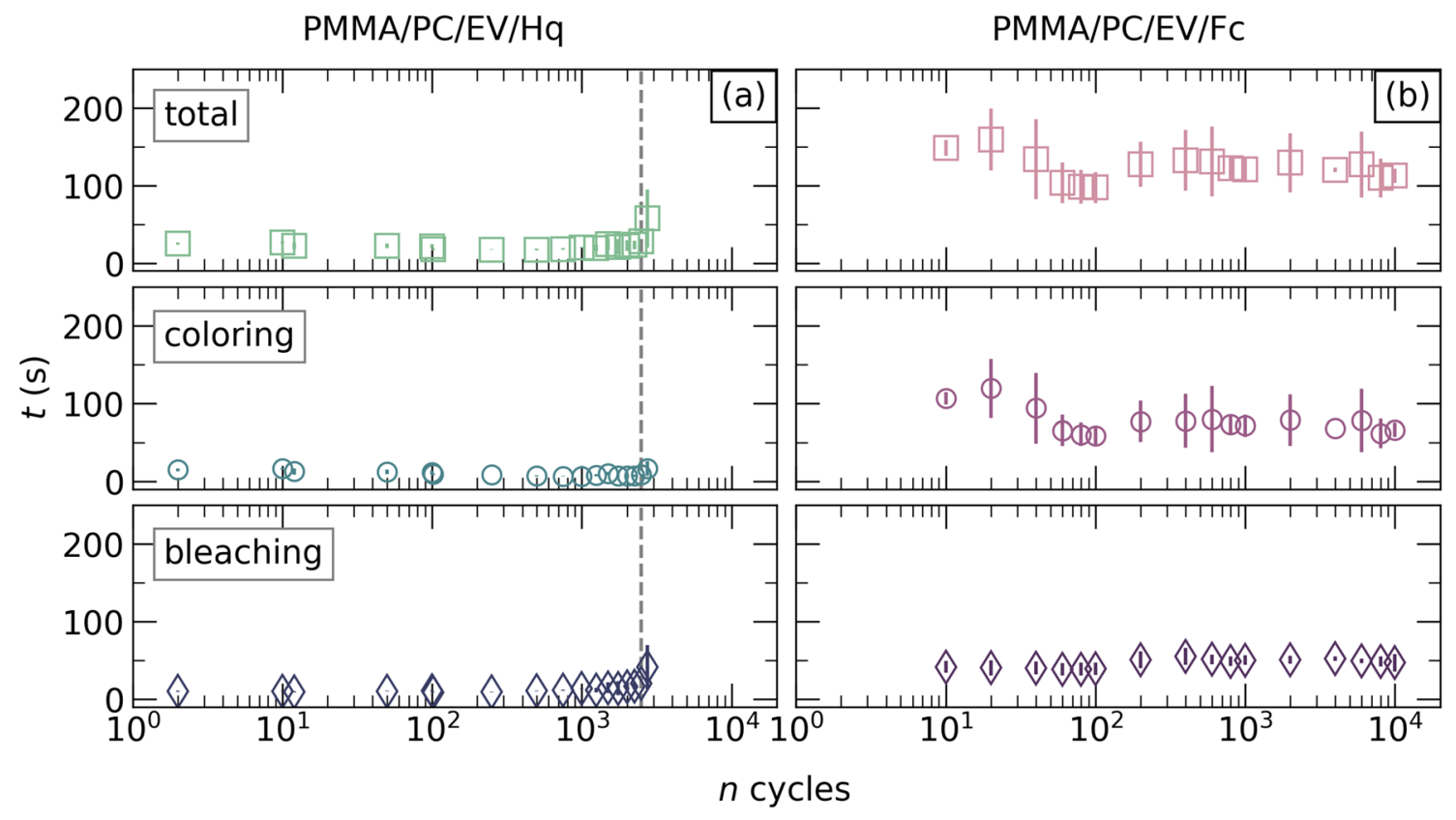

Fig. 4 a(top) Total cycle length, (middle) coloring cycle length, and (bottom) bleaching cycle length for PMMA/PC/EV/Hq samples. Grey vertical line denotes onset of failure. b (top) Total cycle length, (mid- dle) coloring cycle length, and (bottom) bleaching cycle length for PMMA/PC/EV/Fc samples 


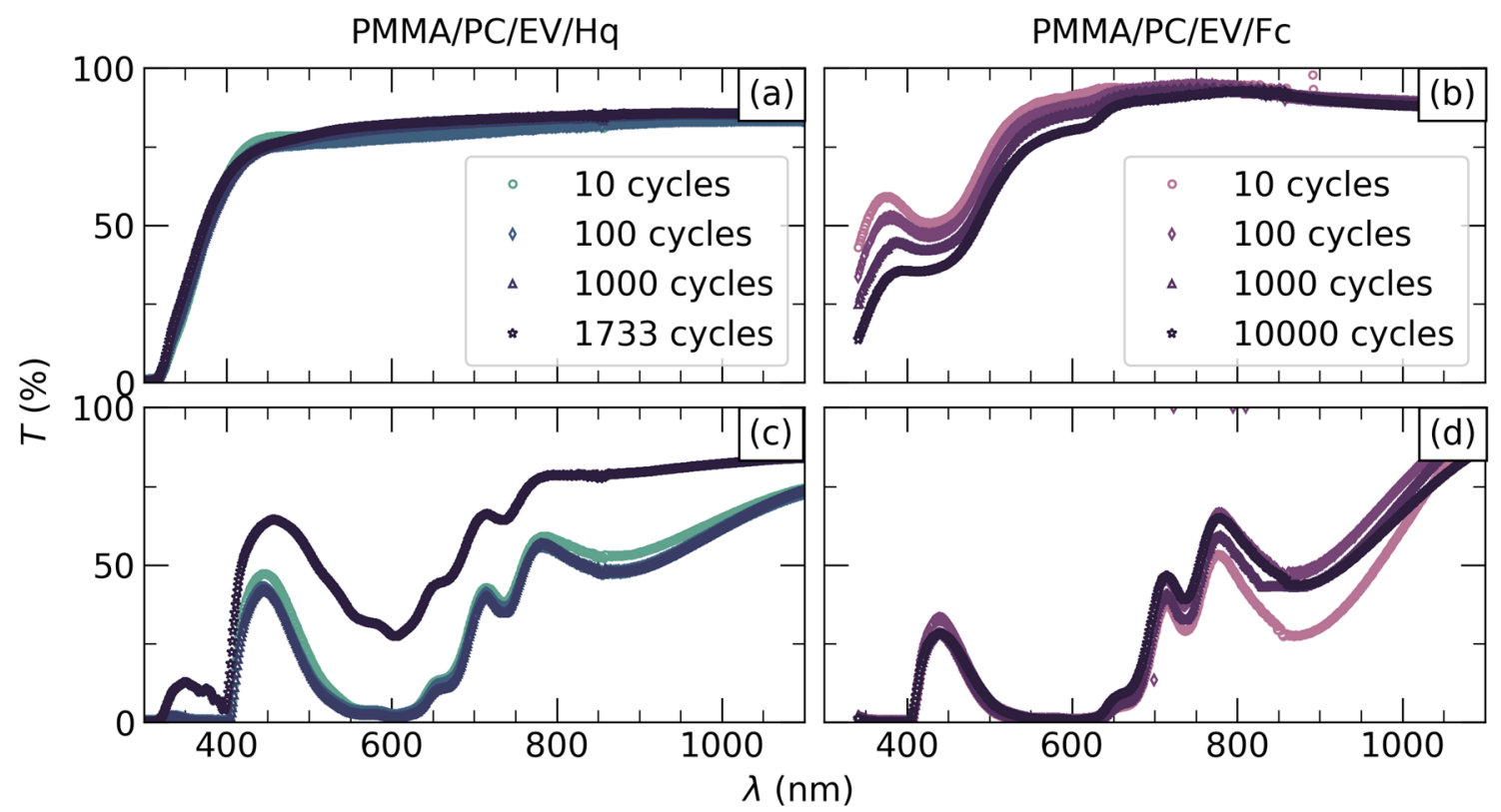

Fig. 5 UV-Vis spectroscopy transmittance spectra of PMMA/PC/EV/ $\mathrm{Hq}$ Sample 3 in the bleached (a) and colored (c) states at 1 cycle, 10 cycles, 100 cycles, 1000 cycles, and at failure (1733 cycles). UV-Vis

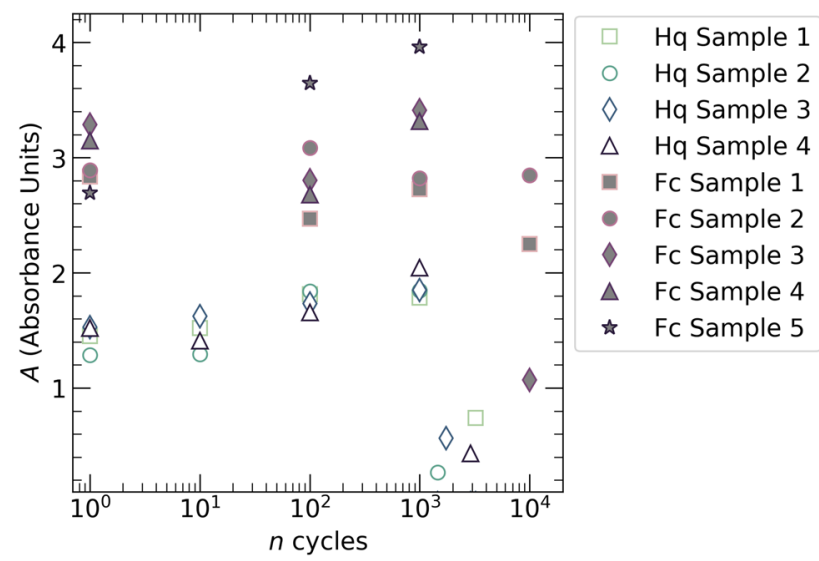

Fig. 6 UV-Vis spectroscopy absorbance data in the colored state at $605 \mathrm{~nm}$ for PMMA/PC/EV/Hq Samples 1-4 at 1 cycle, 10 cycles, 100 cycles, 1000 cycles, and at failure as well as PMMA/PC/EV/Fc Samples $1-5$ at 1 cycle, 10 cycles, 100 cycles, 1000 cycles, and 10,000 cycles

relationship with cycle number, there is a statistically significant relationship between the bleaching length and cycle number. Bleaching length slightly increases with cycling. Comparison of Fig. $4 a$ and $b$ illustrates the trade off between cycling speed and material longevity. While the $\mathrm{Hq}$ samples can transition much more quickly than the Fc samples, the Fc samples survived significantly more cycles than the Hq samples. spectroscopy transmittance spectra of PMMA/PC/EV/Fc Sample 3 in the bleached (b) and colored (d) states at 10 cycle, 100 cycles, 1000 cycles, and 10,000 cycles

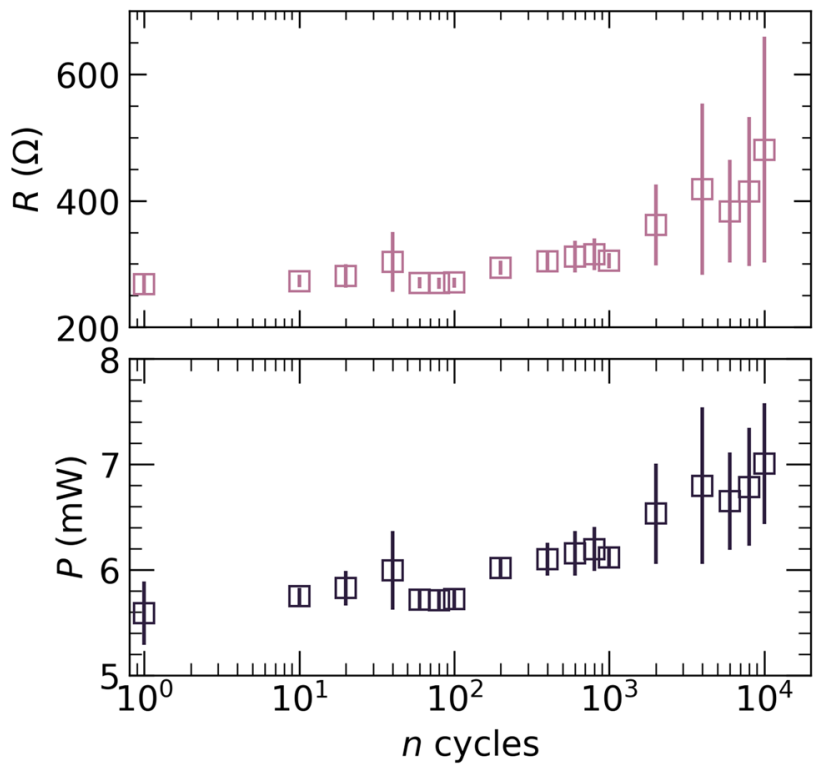

Fig. 7 (top) Maximum sample resistance values during cycling for PMMA/PC/EV/Fc sample 1. (bottom) Maximum power drawn by sample during cycling for PMMA/PC/EV/Fc sample 1

Figure 6 shows the absorbance at $605 \mathrm{~nm}$ for the Fc samples in the colored state during cycling. There exists a statistically significant relationship between absorbance and cycle number (see analysis below). This relationship may be used in conjunction with the 
Beer-Lambert law to quantify the changes occurring in the EC samples with extensive cycling.

Figure 7 shows the calculated values for the sample resistance and power drawn by the samples during cycling. There is a statistically significant relationship with cycle number, as the resistance of the samples and the power drawn by the samples increase with increasing cycle number.

This increase in resistivity may be due to several factors. There may be an increase in resistivity of the ITO, delamination of the ITO, changes in the EC gel due to evaporation of the solvent or from sample handling. There is evidence from absorbance data that suggests that the concentration of the EV radical cation may be changing with cycle length. The study mentioned in the introduction associated the absorbance peak of $605 \mathrm{~nm}$ with the EV radical cation species [12]. The transmittance spectra for PMMA/PC/EV/Fc Sample 1 as a function of cycle are shown in Fig. $5 b$ and d. The transmittance increases over time in the colored state but decreases in the bleached state. At cycle 10,000 the absorbance in the colored state at $605 \mathrm{~nm}$ is less than that for 1 cycle. This relationship in conjunction with the Beer-Lambert law, $A=\sum_{i} \epsilon_{i} / C_{i}$, where the absorbance, $A$, is related to sample thickness, $l$, the molar absorptivity, $\epsilon$, and concentration of species $i, C_{i}$. With thickness and molar absorptivity constant, the absorbance suggests that the concentration of the EV radical cation species may be decreasing with cycling. A decrease in the concentration of the EV radical cation species would imply a decrease in the conductivity of the system given by $\sigma=\sum i Z_{i} U_{i} C_{i}$ where $Z$ is the charge of an ion $i, U_{i}$ is the ion mobility and $C_{i}$ is the ion concentration. The decrease in conductivity due to concentration change would thus lead to a higher resistance measured across the sample with increasing cycle number.

All of the PMMA/PC/EV/Fc samples tested survived cycling up to 10,000 cycles, confirming our hypothesis that lowering the applied voltage by using Fc as the anodic compound, as opposed to Hq, would increase sample longevity. The PTR values of the Fc samples all exceeded the ASTM 2141 defined failure PTR of 4. The PTR values for Samples 1-3 were 87.2, 343.0, and 39.6 respectively. Digital photographs of both samples are included prior to cycling and after cycling in Fig. 8

Single factor analysis of variance (SFE ANOVA) was performed to examine the possible correlations between overall cycle length, coloring time, bleaching time, number of cycles, maximum absorbance, and power draw during operation. In this analysis the cycle number was considered as the single factor, the treatment was each number of cycles, the response was cycle length (broken up in overall, coloring, and bleaching segments), absorbance, resistance, and power. The treatment effects were calculated as the deviation from the overall mean with the null hypothesis that $H 0: \tau(1)=\tau(2) \ldots=\tau(a)=0$ indicating that changing levels of the factor (cycle number) has no effect on the mean response.

In the PMMA/PC/EV/Hq samples, a $p$ value of $1.17 \times 10^{-3}$ was found for the relationship between overall cycle length and cycling number indicating a statistical significance. For absorbance data in PMMA/PC/EV/Hq samples, the samples themselves were not significantly different but self enhancing randomized block design analysis showed that a significant difference existed between 10 and 100 cycles, but not between 100 and 1000 cycles ( $p$ of $4.6 \times 10^{-8}$ ). In the PMMA/PC/EV/FC samples, a $p$ value of 0.282 was found for the relationship between overall cycle length and cycling number indicating no statistical significance. A $p$ value of $2.68 \times 10^{-6}$ was found for the relationship between cycling time and cycling number indicating a statistical significant with over $99 \%$ confidence. Similarly, a $p$ value of $2.52 \times 10^{-10}$ was found for the correlation between power draw during cycling and cycle number indicating a statistical significant with over $99 \%$ confidence.

\section{Conclusion}

Various ethyl viologen electrochromic samples were constructed using different anodic compounds, hydroquinone and ferrocene. These samples were cycled for 10,000 cycles or until failure with different applied voltages. The hydroquinone samples, which were driven at a higher voltage, failed between approximately 1000 and 3000 cycles, whereas all of the ferrocene samples tested survived 10,000 cycles and achieved PTR values well over the ASTM standard for electrochromic failure. The resistance of the electrochromic samples, as well as the power they draw, increased with increasing cycle length, which may be caused by the reduction of the ethyl viologen radical cation concentration during cycling. 
a) Initial samples prior to cycling. PMMA/PC/EV/Fc on left, $\mathrm{PMMA/PC/EV/Hq} \mathrm{on} \mathrm{right}$
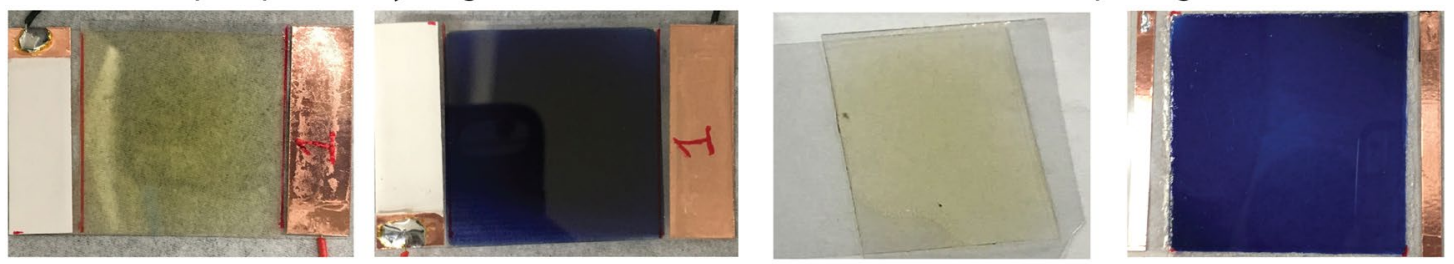

b) $\mathrm{PMMA} / \mathrm{PC} / \mathrm{EV} / \mathrm{Hq}$ samples at failure

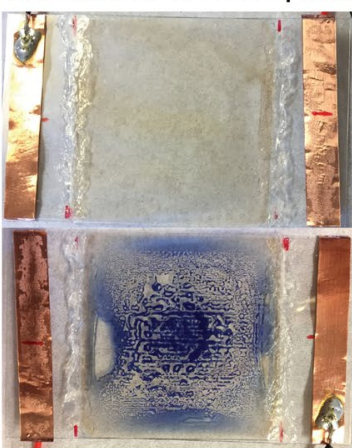

Sample 1: cycle 3,215

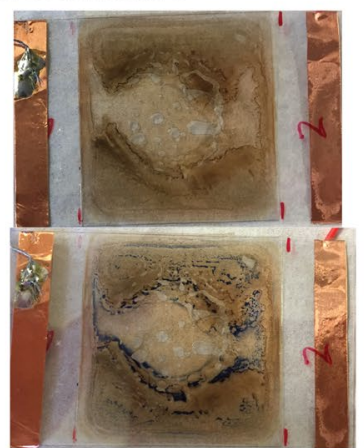

Sample 2: cycle 1,457

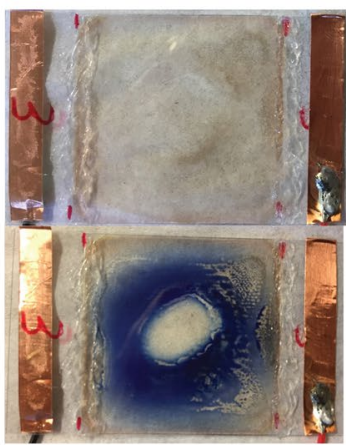

Sample 3: cycle 1,733

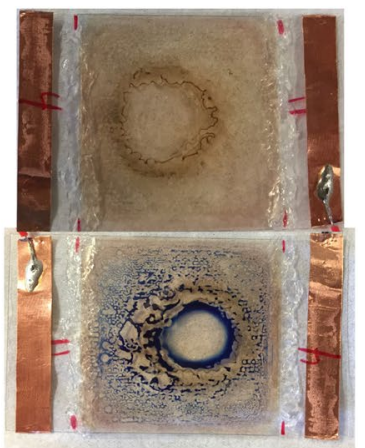

Sample 4: cycle 2,896

c) PMMA/PC/EV/Fc sample after cycling

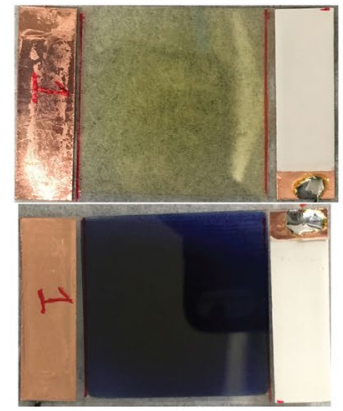

Sample 1: cycle 1

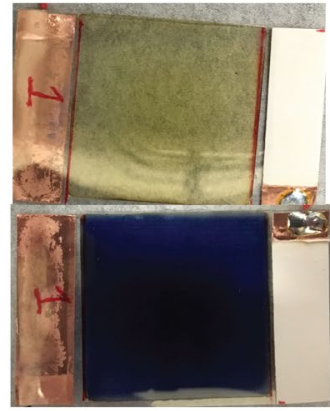

Sample 1: cycle 100

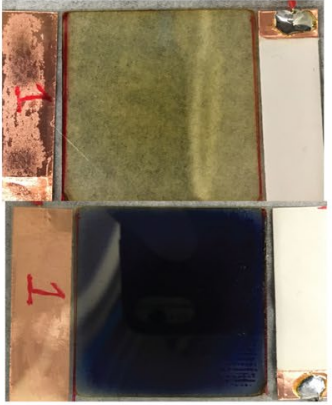

Sample 1: cycle 1,000

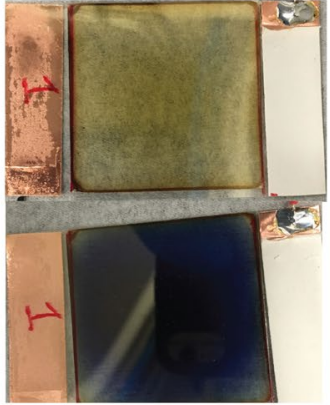

Sample 1: cycle 10,000

Fig. 8 a Samples prior to cycling (PMMA/PC/EV/Fc on left, PMMA/PC/EV/Hq on right). b PMMA/PC/EV/Hq samples at failure (cycle number for each sample shown below). c PMMA/PC/EV/Fc samples after cycling (Sample 1 at various cycles)

Acknowledgements This work was financially supported by the state of Utah through the USTAR 2015 Governor's Energy Leadership Scholars, the USTAR 2016 Industrial Partnership Program, and the Office of Undergraduate Research through the Undergraduate Research Opportunities Program at the University of Utah. We are grateful to Nic Flinner and Max Gallant for their contributions to the development of materials and methods, to Alexander Szendrei for his contribution to the development of the experimental equipment, and to Anton Oliynyk for helpful edits.

\section{Declaration}

Conflict of interest The authors acknowledge that they have a financial and/or business interest in e-Chrome a company that may be affected by the research reported in the enclosed paper.

Open Access This article is licensed under a Creative Commons Attribution 4.0 International License, which permits use, sharing, adaptation, distribution and reproduction in any medium or format, as long as you give appropriate credit to the original author(s) and the source, provide a link to the Creative Commons licence, and indicate if changes were made. The images or other third party material in this article are included in the article's Creative Commons licence, unless indicated otherwise in a credit line to the material. If material is not included in the article's Creative Commons licence and your intended use is not permitted by statutory regulation or exceeds the permitted use, you will need to obtain permission directly from the copyright holder. To view a copy of this licence, visit http://creativecommons. org/licenses/by/4.0/.

\section{References}

1. Monk PM, Mortimer RJ, Rosseinsky DR (2008) Electrochromism: fundamentals and applications. Wiley, London

2. Nishikitani Y, Uchida S, Asano T, Minami M, Oshima S, Ikai K, Kubo T (2008) Photo-and electrochemical properties of linked 
ferrocene and viologen donor-acceptor-type molecules and their application to electrochromic devices. J Phys Chem C 112(11):4372-4377

3. Leventis N, Chung YC (1998) Complementary surface confined polmer electrochromic materials, systems, and methods of fabrication therefor, uS Patent 5,818,636 (Oct. 6 1998)

4. Tsutsumi H, Nakagawa Y, Tamura K (1995) Single-film electrochromic devices with polymer gel films containing aromatic electrochromics. Sol Energy Mater Sol Cells 39(2-4):341-348

5. Platt JR (1961) Electrochromism, a possible change of color producible in dyes by an electric field. J Chem Phys 34(3):862-863

6. Mortimer RJ (1997) Electrochromic materials. Chem Soc Rev 26(3):147-156

7. Green M (1996) The promise of electrochromic systems. Chem Ind 17:641-644

8. Goddard N, Jackson A, Thomas M (1983) Spectrelectrochemical studies of some viologens used in electrochromic display applications. J Electroanal Chem Interfacial Electrochem 159(2):325-335
9. Wang N, Kähkönen A, Damlin P, Ääritalo T, Kankare J, Kvarnström C (2015) Electrochemical synthesis and characterization of branched viologen derivatives. Electrochim Acta 154:361-369

10. Mortimer RJ (1999) Organic electrochromic materials. Electrochim Acta 44(18):2971-2981

11. ASTM International (2014) E2141-14 standard test method for accelerated aging of electrochromic devices in sealed insulating glass units. PA; ASTM International, West Conshohocken

12. Chidichimo $G$, Imbardelli $D$, De Simone $B C$, Barone $P$, Barberio M, Bonanno A, Camarca M, Oliva A (2010) Spectroscopic and kinetic investigation of ethyl viologen reduction in novel electrochromic plastic films. J Phys Chem C 114(39):16700-16705

13. Ho K -C, Hu C-W, Varley TS Electrochromic devices based on metal hexacyanometallate/viologen pairings. Electrochrom Mater Devices

Publisher's Note Springer Nature remains neutral with regard to jurisdictional claims in published maps and institutional affiliations. 